\title{
Historia de la enseñanza de chino como lengua extranjera en China
}

\author{
A history of teaching Chinese \\ as a foreign-language in China
}

\author{
ISABEL MARÍA BALSAS UREÑA*
}

Resumen: Los análisis de la historia de la enseñanza de lenguas extranjeras han sido tradicionalmente realizados por Occidente, por lo que se ha excluido la enseñanza de lenguas asiáticas. El chino no ha sido una excepción a pesar de las particularidades de su didáctica como lengua extranjera, como la estrecha vinculación con la política diplomática del momento o la visión estructuralista que llega hasta la actualidad, inexistente en las metodologías occidentales y que condiciona la forma en que el estudio de esta lengua se expande por el mundo. En este artículo se analiza esta historia, se describen las características de la didáctica en las diferentes épocas, se sugieren conexiones entre ésta y eventos del momento (y viceversa) y se sugieren posibles vías de desarrollo, obtenidas a través de los paralelismos encontrados a lo largo de su trayectoria histórica.

Palabras clave: enseñanza de chino; historia de la enseñanza; chino como lengua extranjera; didáctica del chino; metodologías de la enseñanza de chino.

Abstract: The history of foreign-language teaching has been traditionally analyzed from a Western perspective, thus excluding the teaching of Eastern languages. Chinese is no exception, even though the particularities of its teaching as a foreign language cannot be found

Recepción: 14 de octubre de 2017. / Aceptación: 8 de febrero de 2018.

*Universidad de Granada, sbalsas@ugr.es 
in the teaching of European languages. We can refer, for example, to its close links to topical diplomatic policies and interpretation of structuralism that continues to the present day and influences how this language is studied around the world. This paper explores this history, with a description of every period's didactic approach; it proposes some connections between teaching and historical events (and vice versa), and possible paths for development, stemming from the historical parallelisms detected.

Key words: Chinese teaching; teaching history; Chinese as a foreign language; didactics of Chinese; Chinese teaching methodologies.

\section{Introducción}

La didáctica de lenguas extranjeras busca, mediante el análisis y la reinterpretación de las aportaciones de la lingüística, la psicología, las ciencias de la educación y de la comunicación, generar herramientas que expliquen y faciliten el aprendizaje lingüístico. Es una disciplina dinámica, permeable a los cambios y la evolución de estas materias, de ahí la importancia de comprender tanto las características que han definido los diferentes movimientos didácticos como el proceso de transición que ha llevado de unos a otros. El relato actual de esta evolución, sin embargo, está incompleto, ya que la narración se ha hecho esencialmente desde Europa y Estados Unidos, y se ha obviado la trayectoria de la disciplina fuera de Occidente.

El chino no ha sido una excepción. Su metodología de enseñanza se basa en la noción de integración, que permite que teorías enfrentadas en la didáctica occidental, como el aprendizaje natural propuesto por los métodos directos y el aprendizaje conductista del método audiolingual, tengan cabida simultáneamente en el aula, en una visión ecléctica, productiva y funcional que no se ha dado ni se conoce en la didáctica de lenguas occidentales. El que la literatura sobre la cuestión se encuentre redactada principalmente en chino tampoco ha facilitado el acceso a este conocimiento, útil tanto para la enseñanza de lenguas como para la sinología en el sentido más general del término. 
Uno de los posibles análisis de esta historia, realizado sobre todo con base en fuentes chinas, es el que se muestra a continuación. Con él se pretende facilitar la comprensión del contexto de la enseñanza actual del chino, así como contribuir a generar una visión menos occidentalizada de la didáctica de lenguas. El análisis ha puesto de relieve, además, paralelismos entre épocas pasadas y el momento presente, lo que a su vez ha permitido sugerir vías prospectivas de desarrollo de la disciplina.

\section{Etapas históricas en el desarrollo de la enseñanza de CLE}

El objeto de estudio principal de la historia de la enseñanza de chino como lengua extranjera (en lo sucesivo, CLE) es la metodología, concepto que en China es más amplio que en Occidente e incluye cuestiones como objetivos curriculares, técnicas y procedimientos de clase, evaluación, diseño y explotación del manual, diseño curricular o distribución horaria curricu$\operatorname{lar}(\mathrm{Ma}, 2004$, p. 18).

Siguiendo la clasificación original de Lü Bisong (1990), la mayor parte de los autores establece cuatro etapas en la historia de la didáctica en China: desde los primeros registros hasta la fundación de la República Popular China (1949), etapa precientífica en la que la enseñanza de CLE se realizaba de forma similar a la del chino como lengua materna; una segunda etapa, desde 1949 hasta la finalización de la Revolución Cultural (1976), influida por el método audiolingual y su visión del aprendizaje de lengua como conjunto de estructuras manipulables mediante la conducta; una tercera etapa, durante las décadas de 1980 y 1990, caracterizada por un fuerte desarrollo en la investigación que llevó a la estabilización definitiva de la disciplina; y la última, durante el presente siglo, caracterizada por la diversidad de intereses de investigación y didácticos. 


\section{Primera etapa: desde los primeros registros hasta la fundación de la República Popular China}

La documentación de esta primera etapa es muy escasa. Se sabe que, durante la dinastía Han del Este (206 a.e.c.-9 e.c.), en torno al 66 a.e.c., se fundó la Escuela para los Jóvenes Nobles de las Cuatro Familias para los hijos de los oficiales de origen no chino (Fu, citado por Li, 2015, p. 357) y, debido al contacto comercial entre China y otros países, y al movimiento de religiosos budistas, cabe imaginar que hubo algún tipo de sistema de enseñanza, probablemente similar al que se seguía para enseñar el chino como lengua materna (Wang y Ruan, 2016, p. 6). Se sabe también que durante la dinastía Tang (618907 e.c.) la instrucción tuvo fines de expansión cultural: se acogía y sufragaba a estudiantes de países colindantes (Japón, los tres reinos de Corea, India, Vietnam), los cuales aprendían chino con las obras clásicas en un periodo de entre seis y nueve años (Wang, 2013, p. 31) y, tras aprobar un examen, accedían al estudio de diversas disciplinas para, al finalizar los estudios, regresar a sus países con el conocimiento adquirido en y sobre China. Durante la dinastía Yuan (1206-1368), la política de expansión de fronteras minimizó el interés en la materia; se sabe que hubo un programa de chino para diplomáticos rusos que probablemente funcionó hasta comienzos de la dinastía Qing (1644-1912) (Wang, 2013, p. 32), pero más allá de esto, con lo único que se cuenta es con los materiales que los misioneros occidentales producían para su propio aprendizaje. A mediados del siglo XVIII, con la imposición del Sistema de Cantón (1757-1842), con el que el gobierno imperial frenó las relaciones comerciales entre China y Europa, el interés ya fue prácticamente nulo.

Del análisis de estos primeros registros se desprende un modelo de enseñanza de CLE eminentemente instrumental que, metodológicamente, imitaba los modelos usados en la enseñanza de chino como lengua materna. Se basaban en la memorización de diccionarios de caracteres o de textos clásicos de los que no se analizaba el significado, con los caracteres y la pronunciación por separado. Se sabe, no obstante, que en la enseñanza de chino como lengua materna se buscó facilitar 
este aprendizaje (Tao y Qian, 2012) mediante la sustitución de estos diccionarios, como el Shizhoupian (史籀篇) de la dinastía Zhou (siglo XI-256 a.e.c.), por formatos más accesibles, como los poemas del Libro de los mil caracteres (千字文) de las dinastías Wei-Jin del Sur y del Norte (420-589 e.c.); es posible que para la enseñanza de CLE también se usaran estos libros, aunque no se ha encontrado documentación ni en favor ni en contra de esta hipótesis.

Las siguientes referencias pertenecen ya a los años de la República de China (1912-1949), época en la que la debilidad de la economía no permitió el desarrollo de la disciplina. Se sabe que en la Yenching University, institución americana privada en aquel momento, hubo un grupo de 12 estudiantes de chino que aprendían, además de los textos clásicos, lengua escrita y oral de manera integrada, lo que en el momento supuso una novedad. Cuando en 1925 esta universidad se convirtió en la Yenching School of Chinese Studies, sus 100 profesores y más de 1500 estudiantes seguían el método directo (Wang y Ruan, 2016, p. 119), centrado en el aprendizaje oral de vocabulario y de estructuras de uso cotidiano, lo que contrasta con la situación fuera de esta institución, donde las reflexiones sobre la importancia de la lengua oral, así como cuestiones como la dificultad que entrañaba el aprendizaje de caracteres, apenas comenzaban (Zhao, 2010). También hay constancia de que China mandó a escritores y académicos de renombre, como Wei Jiangong, Lao She, Xiao Gan o Cao Jinghua, para ejercer como profesores de CLE en diferentes países de Asia, África y Europa entre las décadas de 1920 y 1950, aunque no hay estadísticas precisas (Wang, 2003, p. 9; Zhao, 2010). En 1925 se publicó el considerado primer libro para la enseñanza de CLE, el “Curso de fonografía de la lengua nacional” (国语留声机 教程), de Zhao Yuanren.

Durante los años de la guerra civil china (1945-1949) y de la Segunda Guerra Mundial, el foco de desarrollo del CLE se trasladó a Estados Unidos, donde la necesidad de acelerar las comunicaciones llevó a las fuerzas de defensa a contratar a un

\footnotetext{
${ }^{1}$ En el presente artículo se usan las cursivas para los títulos de obras que cuentan con traducción publicada o acuñada, y las comillas en las traducciones propias.
} 
grupo de expertos chinos para que desarrollaran un método de enseñanza de CLE más eficaz. La propuesta de este grupo, dirigido por Zhao Yuanren, estaba fuertemente influida por el audiolingüismo: los alumnos asistían a una hora de instrucción gramatical en inglés y tres de práctica, con nativos o con grabaciones; además, en lugar de caracteres chinos se usó el sistema de romanización Yale, diseñado para angloparlantes, todo lo cual permitió que los alumnos adquirieran una competencia adecuada en medio año, aproximadamente (Zhao, 2010).

\section{Segunda etapa: desde 1949 hasta finales de la Revolución Cultural}

Con la fundación de la República Popular China (1949) se retomó la política de envío de lingüistas de renombre al exterior como forma de establecer vínculos diplomáticos; el primero de ellos, Zhu Dexi, llegó en 1952 a la Universidad de Bulgaria. Dentro de China, se decidió que la encargada de la formación de los estudiantes extranjeros en este nuevo periodo fuera la Universidad de Qinghua. En 1951 llegaron los 33 primeros alumnos, pertenecientes al llamado "Grupo de estudiantes de intercambio de chino de Europa del Este”, que debían cumplir como requisito hablar inglés o ruso para recibir adecuadamente la instrucción (Wang, 2013, p. 34). El diseño del curso fue responsabilidad de Deng Yi, ayudante de Zhao Yuanren en Estados Unidos de 1943 a 1948 ("Académicos destacados de la Universidad de Beijing, la Universidad de Qinghua y la Universidad de Yanjing", 2016). Deng Yi siguió un método similar al usado en Estados Unidos, aunque la gramática se explicaba también en chino y la traducción al inglés se usaba sólo para explicar el significado, con una combinación de elementos de los métodos de gramática-traducción, directo y audiolingual. Con el fin de aceptar más estudiantes, se abrió un curso paralelo en la Universidad de Beijing, donde el profesor Zhou Zumo usaba lo que él describía como "método mixto", una combinación de los métodos directos y de gramática-traducción que permitía a los alumnos, de acuerdo con su creador, ser capaces de entender las lecciones en chino tras un mes de aprendizaje 
(Zhou, citado por Lu y Zhao, 2010, p. 120). En la Universidad del Pueblo también se usó una combinación del método directo con el comparativo soviético, que articulaba la enseñanza en cinco periodos de organización de la clase, repaso de contenidos, explicación de nuevos elementos, prácticas y asignación de tareas para la siguiente clase, pero los recursos que este último requería restringieron su implantación (Zhu, 2010, p. 63). Se sabe, además, que se transitó al uso de caracteres simplificados $\mathrm{y}$ del recién creado sistema de transliteración pinyin (Wang y Ruan, 2016, p. 13), establecido con base en el alfabeto latino; esta decisión revela la importancia que se otorgó a la enseñanza de CLE, ya que la labor de estandarización de la escritura apenas había comenzado. ${ }^{2}$

En 1960, el Instituto de Lenguas Extranjeras de Beijing (actual Universidad de Lenguas Extranjeras de Beijing) creó una oficina de admisión de estudiantes extranjeros que dos años más tarde se fusionó con su homóloga de la Universidad de Beijing para constituir el Centro de Preparación para Estudios Superiores para Estudiantes Extranjeros. En 1964, este Centro se convirtió en el Instituto de Idiomas de Beijing (actual Universidad de Lengua y Cultura de Beijing), única institución dedicada exclusivamente a la investigación y la enseñanza de CLE (Wang, 2003, p. 9). Otras entidades crearon en ese momento programas de formación de alumnos y profesorado: las cifras hablan de 3312 estudiantes de más de 60 países aprendiendo chino en 20 centros de enseñanza superior en 1965, y de unos 100 docentes de CLE entre 1961 y 1966 (Wang y Ruan, 2016, p. 12).

La metodología durante la década de 1960 fue continuista: Li Peiyuan (citado por Zhao, 2010) describía la enseñanza de la época como "basada en un método mixto y centrada en principios de tipo práctico", en la que se combinaban los elementos más eficientes de los métodos de gramática-traducción, directo

${ }^{2}$ El “Primer listado para la ordenación de caracteres variables” (第一批异体 字整理表), primer trabajo de estandarización de la escritura de la Nueva China, se publicó en 1955, aunque fue objeto de numerosas modificaciones en los siguientes años hasta ser remplazado por el “Catálogo de caracteres simplificados” (汉字简化方 案), de 1964, que a su vez fue sustituido por los catálogos de 1977 y 1985 . El catálogo vigente, “Listado de caracteres estandarizados” (通用规范汉字表), es de 2013. 
y audiolingual. En las clases había dos profesores - uno encargado de las explicaciones y otro, de repasar y practicar- que usaban manuales con textos en forma de diálogo y gran cantidad de drills; se practicaba también la lectura intensiva, necesaria para los alumnos que quisieran acceder a cursos universitarios (Lu y Zhao, 2010, p. 120). La base teórica, no obstante, era poco firme, ya que se asentaba en las conclusiones a las que se llegaba tras la práctica con los alumnos (Ren, 1994, p. 91). De este modo, los profesores tenían la gran responsabilidad de determinar qué enseñar y cómo hacerlo (Zhu, 2010, p. 63), lo que llevó a reflexionar sobre la necesidad de la formación del profesorado. Se acordó que los profesores de CLE necesitaban dominar la lengua de sus alumnos para enseñarles adecuadamente; bajo esta premisa se estableció en 1961 un programa de formación de idiomas para futuros profesores que, al revelarse insuficiente, fue sustituido en 1964 por un programa de formación integral (Wang, 2013, p. 35).

La investigación, escasa, seguía modelos básicos de análisis o comentarios de experiencias de aula; se realizaron algunos experimentos con drills durante la década de 1960, pero los resultados no llegaron a recogerse en informes (Sun, 2009, p. 47). En cuanto a los materiales de enseñanza, en 1958 se publicó el primer manual de enseñanza tras la fundación de la República Popular de China, el “Manual de chino" (汉语 教科书), al que le siguió, en 1961, el de “Chino básico" (基础 汉语), más complejo, sistemático y centrado en la práctica y en el uso de las formas cotidianas (Wang y Ruan, 2016, p. 13). Durante los años de la Revolución Cultural (1966-1976), China vetó el contacto con Occidente y, por extensión, la enseñanza de CLE. Aunque las políticas se suavizaron con el regreso de China a la ONU en 1971 y el reconocimiento del chino como una de las lenguas oficiales de esta organización, el aislamiento académico sufrido durante cerca de una década acabó pasando factura. Así, cuando en 1973 el Instituto de Lenguas de Beijing reabrió sus puertas, se regresó a metodologías usadas 10 años antes, ya que localmente no había habido innovación y las teorías didácticas surgidas en Occidente, como los enfoques humanísticos (respuesta física total, método silencioso, sugestopedia...) y el funcionalismo y su interpretación 
pragmática de la lengua, apenas comenzaban a conocerse. La comunidad académica hizo un importante esfuerzo por integrar cuanto antes esas nuevas ideas; de ahí surgió el programa estructural-contextual-funcional, que seguía las teorías de Lü Bisong de que es posible integrar estructura, significado y función, dificultades gramaticales y necesidades comunicativas de los aprendices (Lu y Zhao, 2010, p. 121), en una amalgama teórico-práctica que en Occidente no se dio, pues las nuevas teorías habían sustituido a las anteriores. La investigación trasladó su interés de la gramática comparativa al análisis de errores, ocupación en la que estuvo plenamente centrada en la siguiente etapa (Zhang, 2016, p. 67). Surgieron también las primeras revistas especializadas, como "Enseñanza e investigación de lenguas” (语言教学与研究), que desde los primeros números contó con la colaboración de lingüistas reconocidos como Lü Shuxiang, Zhu Dexi o Lin Tao.

Desde finales de la década de 1970, el incremento del interés por el estudio del chino es patente: la cifra de 383 estudiantes en 1973 prácticamente se decuplicó al cabo de cinco años, lo que a su vez llevó a la apertura de programas de formación del profesorado en 1978 (Wang y Ruan, 2016, p. 13), y a elevar la petición de que la enseñanza de CLE recibiera la consideración de campo de estudio independiente. Se abrieron también los primeros programas de estudios de corta estancia, especialmente con programas de verano (Wang, 2013, p. 36), en respuesta a las demandas de un nuevo perfil de estudiante, que no tiene interés en cursar estudios superiores en China, sino en aprender la lengua.

\section{Tercera etapa: décadas de 1980 y 1990}

Durante la década de 1980, el asentamiento de las ideas importadas y de lo generado localmente favoreció la creación de marcos teóricos más estructurados y sistemáticos (Ren, 1994, p. 95), que a su vez redundaron en una enseñanza más estable. La principal novedad respecto a periodos anteriores fue la decisión de establecer asignaturas centradas en destrezas para los cursos de lengua, lo que permitió su práctica focalizada y, en 
consecuencia, experimentar con diferentes formas de práctica y ejercicios y favorecer la creación de materiales específicos para las parejas auditivo-oral y lectura-redacción (Zhao, 2010). Sin embargo, en la teoría de la enseñanza apenas se realizaron cambios, y se siguió usando una combinación de elementos de los métodos audiolingual, directo y gramática-traducción, con la aparición puntual de algún elemento del enfoque comunicativo de la época, como la descripción de funciones o ejercicios de práctica no controlada. Cabe sugerir aquí que deben interpretarse con cautela las voces que afirman que el enfoque comunicativo se encontraba plenamente integrado en este momento: si se toman como referencia los currículos chinos de enseñanza de idiomas con más trayectoria que el CLE, como puede ser el inglés, no se aprecia que tuviera presencia real hasta la década de 1990 y, aun así, se presenta también desde una perspectiva ecléctica (Adamson y Morris, 1997, p. 25). Es posible, por lo tanto, relacionar estas voces con una búsqueda de conformidad con el halo de corrección que se atribuye a los métodos occidentales por su mayor tradición en la didáctica (Harvey, 1985, p. 184) más que con una descripción objetiva de la situación.

La relación entre los enfoques de aula con base estructuralista, como el método directo o el audiolingual, y los comunicativos, no obstante, generó en la didáctica local sinergias que no se produjeron en Europa. Esto se debe, por una parte, al prestigio del estructuralismo en China debido a que, en el momento de su entrada, su visión de la lengua como algo plenamente descriptible y analizable en estructuras formales había ayudado a concebir herramientas de análisis que la lingüística china por sí misma no había sido capaz de crear y que, además, tras la pausa producida por la Revolución Cultural, habían seguido siendo útiles tanto para el investigador como para el profesor (Zhu, 2010, p. 40). Por su parte, los principios teóricos y prácticos del enfoque comunicativo, con la visión de la lengua como algo que no admitía descripción formal, el alumno en el eje de la enseñanza y las actividades sin respuestas únicas o de tipo lúdico, entre otras muchas cuestiones, resultaron difíciles de aceptar por profesores y alumnos (Yu, 2001; Hu, 2002; Li, 1984), lo que hizo que el impacto de esta nueva corriente fuera 
mucho menor del que había tenido en Europa y que se limitara a la inclusión puntual en los materiales de algún ejercicio de corte más comunicativo. En este sentido, hay también que añadir que la rápida aceptación del enfoque comunicativo en Europa se debió, en parte, a que su gestación había tenido lugar en un momento de agotamiento metodológico y necesidad de una enseñanza de lenguas más eficiente, y había contado además con apoyo institucional desde el Consejo de Europa (lo que, a su vez, había impulsado la publicación de materiales), circunstancias y necesidades que no existían cuando llegó a China. Es posible, teniendo en cuenta estas condiciones y la capacidad de la didáctica china de integrar elementos de metodologías teóricamente opuestas, que el enfoque comunicativo requiriera de más tiempo para su inclusión en la didáctica que otros métodos de instrucción directa que, por su naturaleza más controlada, habrían encajado más rápido y mejor (Zhou y Li, 2016, p. 115); lo que no parece tan probable, teniendo en cuenta esto último, es que se diera un completo abandono de las metodologías tradicionales en favor de las comunicativas.

En 1984, el Ministerio de Educación reconoció la enseñanza de CLE como campo independiente y especialidad de enseñanza. Esta decisión facilitó la apertura de nuevos programas: en 1986, la Universidad de Beijing y el Instituto de Lenguas de Beijing recibieron autorización para ofrecer maestrías a extranjeros, la primera de las cuales se abrió en ese Instituto dos años más tarde (Wang y Ruan, 2016, p. 14). Sin embargo, donde hubo mayor repercusión fue en la investigación. En agosto de 1985 se celebró en Beijing el "Primer Foro Internacional de Enseñanza de CLE”, en el que se habló de fonética, gramática, vocabulario, caracteres, metodologías de aprendizaje, materiales, evaluación y relación lengua-cultura y lengua-literatura $(\mathrm{Xu}, 2004$, p. 63). A pesar de lo que esta diversidad de temas parezca sugerir, el grueso de la investigación se centró en la estandarización lingüística y el análisis de errores (Sun, 2009, p. 50), especialmente en cuestiones que se consideran características del chino, mientras que el resto de los elementos recibió atención puntual (Zhu, 2010, p. 46). La principal novedad probablemente hayan sido las primeras investigaciones 
sobre enseñanza de la cultura (Ren, 1994, p. 93), que, aunque concebida como elemento separado de la lengua, comenzó a ser objeto de un reconocimiento cada vez mayor, hasta que se llegó al diseño, a finales de la década de 1980, del programa estructural-funcional-cultural, presente aún en muchos de los materiales publicados recientemente (Lu y Zhao, 2010, p. 121). Los materiales del momento, no obstante, se regían aún por el programa estructural-contextual-funcional elaborado en los años setenta que, ya maduro, pudo ser implementado plenamente en los manuales.

La evaluación estandarizada de la lengua comenzó también en esta década. Por encargo del Ministerio de Educación, el Instituto de Lenguas de Beijing publicó en 1988 las "Escalas estandarizadas de nivel de chino y currículum por niveles" (汉 语水平等级标准和等级大纲), y un año después, el “Currículum para el HSK (niveles inicial e intermedio)” (汉语水平考试 大纲 [初、中等]). Paralelamente se comenzó a desarrollar el primer examen estandarizado de chino de nivel inicial-intermedio, el Examen de Nivel de Chino, más conocido por sus siglas en chino HSK.

En 1992 tuvo lugar un importante acontecimiento para la enseñanza de CLE: el simposio "Investigación sobre teoría del aprendizaje de lenguas", organizado por las revistas especializadas “Enseñanza de chino en el mundo" (世界汉语教学), “Lingüística aplicada” (语言文字应用) y “Enseñanza e investigación de lenguas", que permitió destacar las necesidades de la investigación y compartir métodos de investigación básica $(\mathrm{Xu}, 2004$, p. 64). Esto propició, por una parte, una mejora de la metodología de investigación, con la incorporación de técnicas cuantitativas (encuesta, estadística) a las conocidas fórmulas de análisis teórico y descripción de experiencia didáctica $y$, por otra, un cambio en los focos de interés de la investigación. Ésta, aunque seguió muy centrada en el análisis de errores, ahora también intentó proporcionar una explicación más práctica de los resultados obtenidos, y analizó errores en áreas como la fonética, el léxico o la pragmática. A finales de la década, incluso aparecieron estudios sobre procesos de adquisición, cuestiones afectivas, estrategias de aprendizaje o competencia lingüística. 
Los resultados de este cambio se percibieron en la enseñanza a lo largo de esa década. La fonética y la pronunciación fueron perdiendo protagonismo frente a las habilidades comunicativas; aparecieron en el aula la tarea y los materiales auténticos, y ganaron peso los contextos situacionales en el uso de la lengua (Cruickshank y Tsung, 2010, p. 217). Por primera vez, también se sopesó la incorporación de la sociopragmática a la enseñanza, aunque la idea no llegó a trasladarse a la práctica (Díaz, 2016, p. 116).

La formalización de la enseñanza continuó: la figura del profesor se reguló en 1991, en 1993 se ofreció el primer grado de CLE, y en 1999, el primer programa de doctorado al que podían acceder extranjeros, en el Instituto de Lenguas de Beijing. Se formalizó también la enseñanza normalizada, con la aprobación por el Ministerio de Educación de los HSK inicial-intermedio y superior en 1990 y 1993, respectivamente, lo que a su vez fomentó la publicación de currículos oficiales, como el "Currículo por niveles de léxico y caracteres de chino" (汉语水平词汇与汉字等级大纲) de 1992, el “Currículo del HSK (nivel superior)” (汉语水平考试大纲 [高等]) de 1995, el “Currículo por niveles de gramática y escalas estandarizadas de lengua china" (汉语水平等级标准与语法等级大纲) de 1996, y el “Currículo para el HSK (nivel principiante)" (中国汉语水 平考试大纲 [基础]) de 1998.

La preocupación curricular no fue exclusiva del entorno institucional: el "Currículo para la enseñanza de chino como lengua extranjera de nivel inicial (gramática, léxico, funciones y contextos)" (对外汉语教学初级阶段教学大纲 [语法、词汇、 功能、情景], Yang Jizhou, 1999) o el "Currículo de funciones para la enseñanza de chino de niveles intermedio y superior" (对外汉语教学中高级阶段功能大纲, Zhao Jianhua, 1999) son también de la época. Hay que tener en cuenta, no obstante, que el eje de estos programas siguió siendo gramática, vocabulario y caracteres, y que, aunque algunos incluyeron funciones o tareas, ninguno realmente se basó por completo en éstas (Zhao, 2004, p. 32). Este fuerte desarrollo curricular contrasta, por otra parte, con el estancamiento que sufrió la creación de manuales: contenidos poco atractivos, gran cantidad de léxico nuevo y poco reciclaje del anterior, ejercicios escasos y poco 
originales, falta de sistematicidad y continuidad entre niveles, explicaciones gramaticales poco claras y contenidos culturales no adaptados a las necesidades de los aprendices son algunos de los problemas que presentaron (Zhao, 1998), pero que no se veía cómo solucionar.

A finales de los años noventa, la enseñanza de CLE alcanzó la estabilidad. Aunque hubo métodos con cierto alcance, como el basado en caracteres, diseñado para estudiantes de lenguas maternas europeas; el basado en el léxico, apoyado en la psicología cognitiva y en estudios de adquisición de segundas lenguas; o el audiovisual situacional, con elementos del análisis del discurso, el que se estableció como forma por excelencia para la enseñanza de CLE es el conocido como método mixto. Su relevancia es tal que sigue usándose en gran parte de los manuales publicados en China, por lo que su análisis no está de más.

\section{El método mixto}

El desfase en la enseñanza de CLE respecto a otras lenguas extranjeras, producido por su tardío desarrollo teórico y práctico, ha llevado a que muchas de las metodologías usadas hayan encontrado inspiración en la didáctica desarrollada fuera de China (Zhao, 2004, p. 53). Sin embargo, y a diferencia de la tendencia occidental de sustituir una teoría por otra, en China se ha optado por la integración de elementos. El resultado de esta fórmula es el método mixto, integrado o ecléctico, considerado como "característico chino", ya que "se diferencia de las metodologías extranjeras en que éstas se apoyan en una única teoría pedagógica para la construcción de los métodos de enseñanza" (Yuan, 2005, p. 116). Sus orígenes se suelen situar en la década de 1950, aunque el método que diseñara Zhao Renyuan en Estados Unidos ya contenía parte de esta filosofía del eclecticismo y no fue realmente sino hasta los ochenta cuando adquirió sistematicidad.

En el método mixto, los contenidos del programa se organizan por un criterio de dificultad (empezando por la fonética), y se presta más atención a las cuestiones que se con- 
sideran particulares del chino. Los caracteres aparecen desde el comienzo de la formación, aunque no necesariamente ligados a la lengua oral, y se incentiva el uso de la lectura en voz alta y la memorización de textos como estrategias de aprendizaje. La secuencia para las lecciones sigue el paradigma de presentación-práctica-producción: se trabajan la fonética y la traducción del vocabulario, se explica la gramática, se lee y analiza el texto del manual, se realizan ejercicios de drills y de práctica controlada, y se concluye con la práctica semicontrolada. Esta secuenciación se repite tema tras tema en la asignatura de lengua, una clase de lectura intensiva cuyos contenidos se refuerzan en el resto de las asignaturas (comprensión auditiva, caracteres, redacción, lectura extensiva, lengua oral, entre otras). La distribución curricular ha pasado por tres modelos (Yuan, 2005, p. 116): el de explicación-práctica (1973-1980), con el que se dedicaban dos horas a la explicación de contenidos y dos a la práctica, con gran influencia del estructuralismo y del conductismo; el de explicación-práctica en cuatro asignaturas (1980-1986), evolución del anterior con el añadido en el diseño curricular de cuatro materias de apoyo y cierta presencia de elementos más comunicativos; y el de enseñanza separada en destrezas (de 1986 al presente), que mantiene la estructura del periodo anterior, aunque con menor rigidez y mayor inclusión de los resultados de experiencias previas.

El primer manual que se puede asociar a este método es el mencionado "Manual de chino" de 1958. Si se compara con los manuales editados actualmente que siguen el método mixto (por ejemplo, la serie Developing Chinese, Universidad de Lengua y Cultura de Beijing, 2011), puede observarse cómo, a pesar de los ajustes realizados, la teoría del aprendizaje es prácticamente idéntica en la base, y es a nivel de técnica de aula donde el profesor dispone realmente de más opciones.

Entre las mejoras que el método mixto necesitaría realizar se encuentran: una mejor integración y secuenciación entre las diferentes materias, una separación de destrezas menos estricta y una mayor presencia de las conclusiones de las investigaciones recientes (Yuan, 2005, p. 118). En la comunidad de enseñanza de CLE, esta metodología ha generado insatisfacción, y son conscientes de la necesidad de optimizar un método que, 
desde la época de los noventa, no ha evolucionado (Yuan, 2005, p. 115).

\section{Cuarta etapa: siglo XXI}

\section{Evolución de la metodología de enseñanza}

El desarrollo de la enseñanza de CLE en este último periodo está estrechamente ligado al de la economía, ya que es ésta, y no un repentino interés global en la lengua o la cultura chinas, la que lo ha impulsado (Li, 2015, p. 380). Esta circunstancia se refleja en las nuevas tipologías de perfil e intereses de estudio que aparecen y, en respuesta a esto, en la multiplicidad de teorías de enseñanza que se sugieren (Cui, 2010, p. 74). Así, en los últimos 15 años han surgido diversas propuestas teóricas de innovación que, sin embargo, no tienen un pleno traslado práctico. Aunque se admite el potencial de la gramática pedagógica, orientada a promover la comprensión de los aprendices y no su conocimiento técnico, al no haber propuestas prácticas no se ha conseguido sacar la gramática tradicional del aula (Zhu, 2010, p. 41). De igual modo, se ha sugerido la posibilidad de convertir el léxico en el eje de la enseñanza $(\mathrm{Lu}, 2000$; Yang, 2003; Li, 2004), pero las propuestas tampoco presentan la suficiente madurez como para ser llevadas a la práctica (Wu, 2004). El enfoque por tareas empieza a adquirir visibilidad $(\mathrm{Ma}, 2000)$, pero las propuestas se enfrentan tanto a los problemas habituales asociados a este enfoque (la evaluación o la falta de investigación sobre las tareas, diferentes en cada lengua) como a los que presenta su uso específico para el chino (como el excesivo tiempo de preparación que las tareas suponen para el estudiante, entre otras; Bao y Kirkebaek, 2013).

La principal tendencia, al parecer, es aproximarse al terreno intermedio o middle ground, un espacio de trabajo donde los docentes integran nuevas pedagogías y enseñanza tradicional siguiendo una filosofía de adaptación, selección, interpretación y adaptación de elementos de las teorías occidentales en un aula en la que las técnicas tradicionales, basadas en razones históricas, pedagógicas y psicológicas (Harvey, 1985, p. 186), también 
reciben reconocimiento dentro de la filosofía integradora característica de la pedagogía china (Deng, 2011, p. 563). El perfil de profesor que se acerca a este terreno intermedio es el de un docente experimentado, nativo o no (Zhang y Jensen, 2013), que conoce, sin idealizar, las pedagogías occidentales centradas en el alumno, y admite también el valor de la repetición y la memorización de las chinas, examina críticamente ambas y las reinterpreta en su práctica profesional (Li, 2016).

Una novedad en este periodo son las TIC y la extensa oferta de aplicaciones, móviles y en línea, que ofrecen para la enseñanza. Sin embargo, en el caso de la enseñanza de CLE, aún no hay trabajos que propongan cómo integrarlas en el aula (McLaren y Bettinson, 2016, p. 244), lo que las convierte en elementos con potencial por explorar.

En cuanto a la cuestión de la cultura ligada a la lengua, los avances han sido escasos. Se reflexiona sobre cómo el origen cultural de cada alumno afecta su forma de acercarse a la lengua y la cultura chinas y, por ende, se piensa que la planificación integrada de ambas materias puede ser necesaria ( $\mathrm{Zhu}, 2010$, p. 56), pero el paso de esta idea a la práctica es deficiente: en el propio Programa general de enseñanza del idioma chino para extranjeros, editado por Hanban y el Instituto Confucio en 2009 y usado habitualmente en programas de formación de profesorado, se separa entre comunicación y cultura (Scrimgeour y Wilson, 2009). Tampoco los conceptos básicos de sociopragmática, necesarios para la comprensión de elementos lingüísticos, como el funcionamiento de la jerarquía en las relaciones sociales, reciben atención en la práctica (Orton, 2010, p. 161).

La metodología de la enseñanza durante este siglo XXI, como se observa, no ha tenido gran desarrollo y, de hecho, se ha descrito como estancada en las décadas de los sesenta y los setenta, anticuada, monolítica, repetitiva, incapaz de incorporar resultados de investigación de fuera de China, poco creativa y confeccionada sin el suficiente cuidado ( $\mathrm{Ma}, 2004, \mathrm{p}$. 17). Las reflexiones sobre la necesidad de una metodología más efectiva son constantes entre los académicos, pero la falta de apoyo institucional a la innovación dificulta los avances en este sentido (Moloney y Xu, 2016, p. 4). 
La producción científica, en cambio, es muy prolífera, aunque el rápido incremento en la demanda de CLE ha multiplicado los perfiles de aprendizaje y, en consecuencia, las cuestiones que deben examinarse. Los análisis coinciden en la idea de que es necesaria más investigación específica para CLE, especialmente en teorías que favorezcan el diseño de una metodología adecuada para la enseñanza (Wang y Ruan, 2016, p. 24). De manera concreta, se han reclamado más estudios interdisciplinares sobre teorías de aprendizaje que tengan en cuenta las características particulares del chino, realizados con más y mejores métodos de investigación, con más perspectivas en el tratamiento de los contenidos y mayor profundidad en sus análisis (Xu, 2004, p. 70); es necesario conocer más y mejor el contexto y el perfil de los aprendices, la adquisición del CLE y su pedagogía, la aplicabilidad de la lingüística y los instrumentos de evaluación (Cruickshank y Tsung, 2010; Zhao, 2001); y se requiere llevar al aula los resultados que se obtengan $(\mathrm{Ma}$, 2004, p. 17). Se ha llamado la atención, además, sobre la necesidad de mejorar la calidad y no tanto la cantidad (Sun, 2009 , p. 90), ya que abundan los trabajos de tipo general sobre cuestiones ya muy trabajadas, mientras que las investigaciones específicas escasean y en muchas ramas no se cuenta con especialistas; así, morfología, sintaxis o gramática son áreas recurrentes para la investigación en CLE (Zhang, 2016, p. 70), pero escasean trabajos que se ocupen de la sociolingüística, el análisis del discurso o la pragmática. Del mismo modo, apenas se cuenta con investigaciones con alumnos de nivel intermedio y superior (Sun, 2009, p. 50), con las repercusiones que esto puede tener a largo plazo.

En pocas palabras, la enseñanza de CLE en la actualidad se enfrenta al reto del desarrollo de pedagogías, programas y evaluación adecuados a los nuevos contextos de enseñanza, así como a la generación de una mayor conexión entre la lingüística aplicada, la investigación en CLE y la práctica de aula (Lu y Zhao, 2010, p. 128). Mientras se realiza la investigación necesaria para que esto suceda, el profesorado se encuentra acomodado en el enfoque mixto, o bien generando su propio terreno intermedio. Ambas cuestiones, por otra parte, están relacionadas con dos nuevas situaciones que surgen también en 
este periodo: el modelo de enseñanza del Instituto Confucio y el nuevo perfil de profesor.

\section{La influencia del Instituto Confucio}

La Oficina Nacional China para la Enseñanza de Chino como Lengua Extranjera, más conocida por su abreviatura Hanban, realiza una importante labor de expansión de la enseñanza de chino a través de la red del Instituto Confucio. Desde la apertura del primer centro en Seúl en 2005 se han abierto más de 1580 centros en 140 países (http://www.hanban.edu.cn/ confuciousinstitutes/node_10961.htm); entre sus funciones principales se encuentra, ad̄emás de la enseñanza de lengua y la formación de profesorado, la administración del actual modelo del HSK, que desde 2007 sustituye los diseñados en 1988 por la Universidad de Lengua y Cultura de Beijing. Estos nuevos HSK, menos complejos, de orientación más comunicativa, con pruebas orales y de CLE para fines específicos, son la cara más visible de la investigación que, junto con Hanban, también lleva a cabo esta institución; herramientas marco, como los estándares de competencias del profesorado de CLE ("Estándares para profesores de chino como lengua extranjera” 国际汉语 教师标准, 2007 y 2012), de evaluación de lengua (“Escalas de nivel para chino como lengua extranjera” 国际汉语能力标 准, 2007) y de planificación curricular (Programa general de enseñanza del idioma chino para extranjeros, 2009) son también resultado de esta labor.

Aunque el Instituto Confucio da a sus centros libertad para adaptar la metodología a las necesidades de aprendizaje locales, la facilitación y popularización de los HSK ha fomentado un modelo de aprendizaje competitivo, orientado a la obtención de los certificados de nivel de lengua. Esto ha favorecido el incremento del interés en el estudio de chino, pero también ha contribuido a la expansión sistemática del enfoque mixto, ya que el foco descriptivo en la gramática y los elementos léxicos que lo caracterizan facilita la preparación de unas pruebas que están muy orientadas a medir estos dos elementos. Esta situación ha generado pérdida de interés en cuestiones como 
la investigación curricular (Tsung y Cruickshank, 2010, p. 2), además de favorecer el empobrecimiento de la metodología de enseñanza.

\section{Problemáticas ligadas al profesorado}

El perfil tradicional de profesor hasta el momento ha sido el de nativo chino con cierta formación en CLE o el de especialista en áreas tradicionales de la sinología (literatura, traducción...) sin formación en didáctica (Zhou, 2010). Sin embargo, en este momento surgen nuevos perfiles de profesor, como el nativo formado en pedagogía en Occidente o el no nativo formado en Occidente. Se genera así, por primera vez, el debate sobre qué tipo de perfil es el más adecuado, si el nativo con mayor dominio de lengua y conocimiento de su cultura, pero con menor capacidad para la gestión de un aula con alumnos de educación no china, o un no nativo más empático con las dificultades del alumno y eficaz en la gestión del aula, pero con menor capacidad lingüística (Sung y Poole, 2016). La tendencia parece ser la de profesor nativo con educación occidental (Xing, 2006, p. 15), pero las carencias de uno y otro llevan a reflexionar sobre la necesidad de formación del profesorado; de hecho, un informe del National Centre for Languages de Reino Unido de 2007 afirmaba que la falta de profesorado preparado era una de las limitaciones para el desarrollo de la enseñanza de CLE (Orton, 2010, p. 152).

El tipo de formación del profesorado determina, por otra parte, la oferta editorial. La reticencia del profesorado al cambio hace que la innovación se limite a la inclusión puntual, dentro de manuales que siguen el método mixto, de tareas o de ejercicios de role-play, lo que permite al profesor sentir que se encuentra usando materiales novedosos, sin tener realmente que abandonar la zona de confort del método mixto. Se produce así un círculo vicioso: el profesorado no exige nuevos materiales, por lo que las editoriales tampoco se ven forzadas a innovar; al no contar con materiales renovados, aquellos profesores que desean cambiar su forma de trabajo se ven obligados a preparar los suyos propios, cuestión que requiere 
una inversión de tiempo notable y que puede llevarles a decidir no innovar.

En resumen, aunque en esta última etapa en la enseñanza de CLE no hay un punto de ruptura radical respecto a la anterior, sí se percibe la influencia del debate entre la metodología occidental y la china, debate que, por el momento, sólo ha encontrado aplicación a título individual por profesores descontentos con el método mixto que han decidido trabajar desde el terreno intermedio. Se percibe cierta intención innovadora, aislada y difícil de visualizar en conjunto, pero no puede afirmarse que haya una verdadera corriente de renovación en la enseñanza.

\section{Conclusiones}

La enseñanza de CLE ha estado fuertemente influida por los métodos occidentales de gramática-traducción, audiolingual y directo, que se encuentran en el corazón de la metodología considerada propia del chino, el método mixto, y que en las últimas décadas se completa con algunos elementos del funcionalismo y de los enfoques comunicativos. La diversificación de los contextos y los perfiles de aprendizaje ha llevado a que la metodología de enseñanza empiece a atender necesidades particulares, como el sistema de escritura (Cruickshank y Tsung, 2010, p. 218), pero no existe aún una pedagogía específica para el chino, ni va a ser fácil producirla, ya que no se cuenta con la investigación necesaria para ello. Dado que el propio uso de la lengua de los nativos aún está siendo documentado y codificado (Orton, 2010, p. 159), cabe suponer que pasará un tiempo antes de que esta pedagogía se genere.

Mientras tanto, los expertos coinciden en que se debe "construir un sistema de teorías de la enseñanza, modificable y con base en principios que sirvan de guía para la gestión de las interacciones entre la enseñanza/aprendizaje y la gestión de la enseñanza que las conecta”, compuesto de teorías actuales, realistas y completas y por la propia experiencia surgida de la práctica (Ren, 1994, p. 102), y que cuente con una investigación de base lo suficientemente amplia y fuerte como para adaptarse a los diferentes perfiles de aprendiz (Zhao, 
2010). Este enfoque prestaría atención a la estructura y a la función del lenguaje al mismo tiempo, incluiría elementos de la didáctica de dentro y fuera de China e integraría enseñanza de gramática, de caracteres y de caracteres respecto al léxico (Zhu, 2010, p. 50), cuestiones sobre las que apenas se cuenta con propuestas en la actualidad.

Si se compara la situación vigente con otras anteriores, es posible encontrar cierto paralelismo, como el periodo entre finales de la década de 1970 y comienzos de la de 1980, en el que la investigación y la práctica parecían seguir dos rutas diferentes. Es posible que los nuevos elementos en el área que requieren investigación (perfiles de alumno, variedad de objetivos, nuevas tecnologías...) hayan producido tal diversificación en la investigación que hoy resulte imposible percibir las repercusiones. Es igualmente posible que la influencia que ejercen los HSK y la enseñanza estandarizada, ligada al método mixto, sea mayor de lo que parece. Por último, también cabe la posibilidad de que el método mixto esté llegando a una situación de agotamiento de la cual, sin marcos alternativos completos, no puede salir: la cantidad de estudios que reclaman una teoría de la enseñanza y una metodología propias para el chino, argumentada y realista, que incluya todas las dificultades de esta lengua, parecen apuntar en esta dirección.

Dirección institucional de la autora:

Departamento de Lingüistica General y Teoría de la Literatura

Universidad de Granada

Facultad de Filosofía y Letras

Nuevo Edificio Departamental, Planta -1. Despacho L13

Campus Universitario de Cartuja

18071 Granada, España

\section{Referencias}

Académicos destacados de la Universidad de Beijing, la Universidad de Qinghua y la Universidad de Yanjing 北大清华燕京的才子佳人. (29 de febrero de 2016). The China Press 纽约侨报. Recuperado de http://ny.uschinapress.com/weekends/2016/02-29/89860. html 
Adamson, B. y Morris, P. (1997). The English curriculum in the People's Republic of China. Comparative Education Review, 41(1), 3-26. https://doi.org/10.1086/447413

BAo, R. y Kirkebæk, M. J. (2013). Danish students' perceptions of task-based teaching in Chinese. En M. J. Kirkebæk, X. Du y A. Arup Jensen (Eds.), Teaching and learning culture: Negotiating the context (pp. 61-78). Róterdam: Sense Publishers.

Cruickshank, K. y Tsung, L. (2010). Teaching and learning Chinese: A research agenda. En L. Tsung y K. Cruickshank (Eds.), Teaching and learning Chinese in global contexts: Multimodality and literacy in the new media age (pp. 213-224). Londres: Continuum.

CUI, X. 崔希亮 (2010). 汉语国际教育 “三教” 问题的核心与基础 [El problema de "las tres T" en la enseñanza de chino lengua extranjera]. 世界汉语教学 [Enseñanza de chino en el mundo], 1(24), 73-81.

Deng, Z. (2011). Confucianism, modernization and Chinese pedagogy: An introduction. Journal of Curriculum Studies, 43(5), 561-568. https://dx.doi.org/10.1080/00220272.2011.61 7837

DíAz, A. R. (2016). Developing interculturally-oriented teaching resources in CFL: Meeting the challenge. En R. Moloney y H. L. Xu (Eds.), Exploring innovative pedagogy in the teaching and learning of Chinese as a foreign language (pp. 115-135). Singapur: Springer. https://dx.doi.org/10.1007/978-981-287-772-7_7

Harvey, P. (1985). A lesson to be learned: Chinese approaches to language learning. ELT Journal, 39(3), 183-186. https://dx.doi. org/10.1093/elt/39.3.183

Hu, G. (2002). Potential cultural resistance to pedagogical imports: The case of communicative language teaching in China. Language, Culture and Curriculum, 15(2), 93-105. https://dx.doi. org/10.1080/07908310208666636

LI, X. (1984). In defence of the communicative approach. ELT Journal, 38(1), 2-13. https://dx.doi.org/10.1093/elt/38.1.2

LI, X. 李晓琪 (2004). 关于建立词汇 - 语法教学模式的思考 [Reflexiones en torno al enfoque léxico en la enseñanza de lenguas]. 语言教学与研究 [Enseñanza e investigación de lenguas], (1), 23-29.

LI, Y. (2015). Language policies and practices in China: Language planning in China. Berlín: De Gruyter Mouton.

LI, Z. (2016). CFL Teacher identity construction: A core element of future innovative practice. En R. Moloney y H. L. Xu (Eds.), 
Exploring innovative pedagogy in the teaching and learning of Chinese as a Foreign Language (pp. 177-192). Singapur: Springer. https:/dx.doi.org/10.1007/978-981-287-772-7 10

LÜ, B. 吕必松 (1990). 对外汉语教学发展概要 [C̄ompendio sobre el desarrollo de la enseñanza de chino como lengua extranjera]. Beijing: Beijing Language and Culture University Press.

LU, J. 陆俭明 (2000). “对外汉语教学” 中的语法教学 [La gramática en la enseñanza de chino como lengua extranjera]. 语言教学与 研究 [Enseñanza e investigación de lenguas], (3), 1-8.

Lu, J. y Zhao, Y. (2010). Teaching Chinese as a foreign language in China: A profile. En L. Tsung y K. Cruickshank (Eds.), Teaching and learning Chinese in global contexts: Multimodality and literacy in the new media age (pp. 117-130). Londres: Continuum.

MA, J. 马箭飞 (2002). 任务式大纲与汉语交际任务 [El currículo por tareas y la enseñanza de chino por tareas]. 语言教学与研究 [Enseñanza e investigación de lenguas], (4), 27-34.

MA, J. 马箭飞 (2004). 汉语教学的模式化研究初论 [Breve análisis sobre la investigación en metodologías de la enseñanza de chino]. 语言教学与研究 [Enseñanza e investigación de lenguas], (1), 17-22.

McLaren, A. E. y Bettinson, M. (2016). Digital tools for Chinese character acquisition and their impact on student motivation. En R. Moloney y H. L. Xu (Eds.), Exploring innovative pedagogy in the teaching and learning of Chinese as a foreign language (pp. 235-251). Singapur: Springer. https://doi.org/10.1007/978981-287-772-7 13

Moloney, R. y Xu, H. L. (2016). Taking the initiative to innovate: Pedagogies for Chinese as a foreign language. En R. Moloney y H. L. Xu (Eds.), Exploring innovative pedagogy in the teaching and learning of Chinese as a foreign language (pp. 1-17). Singapur: Springer. https://dx.doi.org/10.1007/978-981-287-77271

ORTON, J. (2010). Educating Chinese language teachers. En L. Tsung y K. Cruickshank (Eds.), Teaching and learning Chinese in global contexts: Multimodality and literacy in the new media age (pp. 151164). Londres: Continuum.

REN, Y. 任远 (1994). 对外汉语教法研究的回顾与展望 [Evolución y desarrollo de la investigación en metodologías de la enseñanza de chino como lengua extranjera]. 语言教学与研究 [Enseñanza e investigación de lenguas], (2), 90-103.

SCRIMgeour, A. y Wilson, P. (2009). International curriculum for Chinese language education. Babel, 43(2), 35-38. 
SuN, D. 孙德金 (2009). 五十余年对外汉语教学研究纵览 [Panorama de la investigación en enseñanza de chino como lengua extranjera en los últimos cincuenta años]. 语言教学与研究 [Enseñanza e investigación de lenguas], (2), 45-53.

Sung, K. Y. y Poole, F. (2016). Differences between native and non-native Chinese speaking teachers: Voices from overseas students who study Chinese in China. En C. P. Chou y J. Spangler (Eds.), Chinese education models in a global age (pp. 133-147). Singapur: Springer. https://dx.doi.org/10.1007/978-981-100330-1 10

TAO, L. y Qian, G. (2012). Historical perspectives on Chinese written language and literacy education in China. En C. B. Leung y J. Ruan (Eds.), Perspectives on teaching and learning Chinese literacy in China (pp. 1-17). Dordrecht: Springer. https://doi. org/10.1007/978-94-007-4822-4_1

TsunG, L. y Cruickshank, K. (2010). Emerging trends and issues in teaching and learning Chinese. En L. Tsung y K. Cruickshank (Eds.), Teaching and Learning Chinese in Global Contexts: Multimodality and literacy in the new media age (pp. 1-10). Londres: Continuum.

WANG, D. (2013). Of empire and the city: English in the Chinese foreign language classroom. Berna: Peter Lang.

WANG, L. 王路江 (2003). 从对外汉语教学到国际汉语教学 [De “enseñanza de chino como lengua extranjera” a "enseñanza internacional de chino”]. 世界汉语教学 [Enseñanza de chino en el mundo], (3), 9-12.

WANG, W. y Ruan, J. (2016). Historical overview of Chinese language education for speakers of other languages in China and the United States. En J. Ruan, J. Zhang y C. Leung (Eds.), Chinese language education in the United States (pp. 1-28). Cham: Springer. https://dx.doi.org/10.1007/978-3-319-21308-8_1

Wu, Y. 吴勇毅 (2004). 汉语作为第二语言语法教学的 “语法词汇 化” 问题 [El problema de la lexicalización de la gramática en la enseñanza de chino como segunda lengua]. En J. Tian 田郬 (Ed.), 第七届国际汉语教学讨论会论文选 [Textos escogidos del 70 Simposio Internacional de Enseñanza de Chino como Lengua Extranjera]. Beijing: Peking University Press.

XING, J. Z. (2006). Teaching and learning Chinese as a foreign language: A pedagogical grammar. Hong Kong: Hong Kong University Press.

Xu, Z. 徐子亮 (2004). 对外汉语学习理论研究二十年 [Veinte años de investigación en teorías sobre el aprendizaje de chino como 
lengua extranjera]. 世界汉语教学 [Enseñanza de chino en el mundo], 4(70), 63-73.

YANG, H. 杨惠元 (2003). 强化词语教学, 淡化句法教学 [Fomentar la enseñanza de léxico, reducir la enseñanza de sintaxis]. 语言教学与研究 [Enseñanza e investigación de lenguas], (1), 37-43.

YU, L. (2001). Communicative language teaching in China: Progress and resistance. TESOL Quarterly, 35(1), 194-198. https://dx.doi. org $/ 10.2307 / 3587868$

YUAN, Z. 原载 (2005). 基础汉语教学模式的改革 [Una reforma inicial en la metodología de enseñanza de chino]. En Y. Cui 崔 永华 (ed.), 对外汉语教学的教学研究 [Investigación sobre la enseñanza de las metodologías de enseñanza de chino como lengua extranjera] (pp. 114-123). Beijing: Foreign Language Teaching and Research Press.

Zhang, C. y Jensen, A. A. (2013). Professional identity construction of non-native Chinese language teachers. En M. J. Kirkebæk, X. Du y A. Arup Jensen (Eds.), Teaching and learning culture: Negotiating the context (pp. 113-127). Róterdam: Sense Publishers.

ZhANG, J. (2016). Understanding Chinese as a foreign language from the perspective of second language acquisition. En J. Ruan, J. Zhang y C. Leung (Eds.), Chinese language education in the United States (pp. 63-82). Cham: Springer. https://dx.doi. org/10.1007/978-3-319-21308-8 4

ZHAO, J. 赵金铭 (1998). 论对外汉语教材评估 [Evaluación de materiales para la enseñanza de chino como lengua extranjera]. 语 言教学与研究 [Enseñanza e investigación de lenguas], (3), 4-19.

ZHAO, J. 赵金铭 (2001). 对外汉语研究的基本框架 [Un marco básico para la investigación de chino como lengua extranjera]. 世界汉 语教学 [Enseñanza de chino en el mundo], (3), 3-11.

ZHAO, J. 赵金铭 (2004). 对外汉语教学概论 [Panorama de la enseñanza de chino como lengua extranjera]. Beijing: The Commercial Press.

ZhAO, J. 赵金铭 (2010). 对外汉语教学法回视与再认识 [Nuevas aproximaciones a la metodología de la enseñanza de chino como lengua extranjera]. 世界汉语教学 [Enseñanza de chino en el mundo], (2). Recuperado de http://www.cssn.cn/yyx/ yyx_gwyyx/201401/t20140121949482.shtml

Zhou, M. (2010). Globalization and language order: Teaching Chinese as a foreign language in the United States. En L. Tsung y K. Cruickshank (Eds.), Teaching and learning Chinese in global 
contexts: Multimodality and literacy in the new media age (pp. 131149). Londres: Continuum.

Zhou, W. y Li, G. (2016). Chinese language teachers' pedagogical adjustment and classroom management in cross-cultural contexts. En J. Ruan, J. Zhang y C. Leung (Eds.), Chinese language education in the United States (pp. 211-230). Cham: Springer. https://doi.org/10.1007/978-3-319-21308-8 11

ZHU, Z. (2010). A historical perspective of teaching Chinese as a second language. En J. Chen, C. Wang y J. Cai (Eds.), Teaching and learning Chinese: Issues and perspectives (pp. 33-69). Charlotte, NC: Information Age Pub. 
